\title{
Die logistische Stadt
}

Rezension zu Deborah Cowen (2014): The Deadly Life of Logistics. Mapping Violence in Global Trade. Minneapolis: University of Minnesota Press.

Abb. 1 Titel des Buches (Quelle: University of Minnesota Press

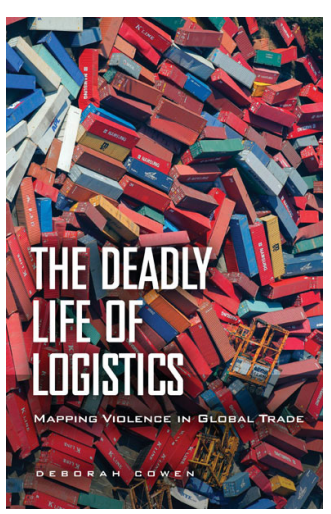

Das Thema Logistik ist im akademischen Betrieb angekommen. Sei es als „logistical hub“ (Sundarakani 2017), als „logistical power“ (Gregson et al. 2017) oder gar als „logistical fix“ des westlichen Nachkriegskapitalismus (Danyluk 2017): Der Begriff der Logistik hat in den vergangenen Jahren in vielfältiger Weise Eingang in sozialwissenschaftliche und humangeographische Forschungskontexte gefunden. Die Orte, die dabei in den Mittelpunkt des Interesses rücken, sind nicht weniger vielschichtig: Containerhäfen und Flughäfen, die gigantomanischen Lagerhäuser von Amazon und Walmart sowie die Alltags- und Arbeitspraxen, die dort stattfinden, bieten reichlich Denk- und Untersuchungsstoff (Danyluk 2017, Moody 2017). Politisch wiederum kristallisieren sich mehrere Debattenstränge heraus: Einerseits geht es darum, die fundamentale Bedeutung logistischer Arbeitsprozesse und-kämpfe für globale Verwertungs- und Ausbeutungsprozesse herauszuarbeiten. Diese Kämpfe, so die Hoffnung, könnten einen entscheidenden politischen Hebel gegen einen Kapitalismus bieten, dessen Produktions- und Zirkulationsprozesse zunehmend auf globaler Ebene stattfinden und somit in entscheidendem Maße auf den reibungslosen Ablauflogistischer Arbeit angewiesen sind (Moody 2017, Transnational Social Strike Platform 2017). Andererseits geht es um die Frage, bis zu welchem Grad die gebaute Umwelt der Logistik aus ihren kapitalistischen Verwertungszwängen herausgelöst und vielleicht sogar in eine postkapitalistische Nutzung überführt werden könnte. Während anarchistische Beiträge dazu tendieren, logistische Infrastrukturen als Schöpfungen des Kapitalismus zu kennzeichnen, denen nicht anders beizukommen sei als durch Sabotage und Zerstörung (The Invisible Committee 2007; 2014), wird von marxistischer Seite durchaus dafür plädiert, genauer über die kommunistische Aneignung logistischer Infrastrukturen nachzudenken (Bernes 2013, Fisher 2013: 96-112, Toscano 2011; 2014). Ähnliches gilt dann auch für Diskussionen rund um die sogenannte ,Smart City'. Auch hier gibt es lohnenswerte Überlegungen dazu, neuartige Technologien und Infrastrukturen (etwa Methoden der Datenerfassung und -analyse, das „Internet der Dinge“), die einstweilen nur den Kapitalinteressen zu dienen scheinen, für emanzipatorische Zwecke einzuspannen (Morozov/Bria 2017, Söderström et al. 2014). 
Es sind Debatten wie diese, denen Deborah Cowen mit The Deadly Life of Logistics. Mapping Violence in Global Trade eine fundierte Grundlage geschaffen hat. Denn ihr Buch liefert einen willkommen nüchternen Ruhepol und zugleich einen analytischen und politischen Kompass für das, was unter dem Stichwort der - Achtung, großes Wort - ,logistischen Revolution' noch zu verhandeln sein wird. Was Cowens Werk von anderen Publikationen abhebt, ist gerade die Sorgfalt und Ausgiebigkeit, mit der sie die konfliktreiche Entstehungsgeschichte der Logistik in bester genealogischer Manier zurückverfolgt. Mit viel Gespür für widerspenstige Details lässt Cowen die zwei historischen Triebfedern der logistischen Revolution hervortreten: die militärische Logistik mit ihren Problemen der Nachschuborganisation und -koordination einerseits, die Wirtschaftslogistik mit ihren Problemen der Kalkulation und Absicherung globaler Lieferketten andererseits. Was sich auf knapp 250 Seiten auftut, ist eine historisch-räumliche Kartographie der Logistik an den „shifting boundaries between ,civilian' and ,military' domains“ (S. 4).[1] Dass Cowen an dieser gewaltvollen Geschichte mit einem extrem vielseitigen Analyseinstrumentarium ansetzt, das kritische Ansätze aus der (Stadt-)Geographie und Kartographie ebenso mit einschließt wie marxistische Theorie, kritische Sicherheitsforschung oder queer-theoretische Überlegungen, macht den nachhaltigen Reiz ihres Buches aus. Kurzum: Cowens Buch liefert fundierte Gründe dafür, den Widersprüchen der Logistik noch sehr viel genauer nachzuspüren, nicht zuletzt - und dazu gleich noch mehr - in ihren stadtgeographischen Dimensionen.

Logistik, so Cowens Kernthese, ist in ihrer Bedeutung für den heutigen Kapitalismus kaum zu überschätzen. Dieser, so argumentierte schon Karl Marx im zweiten Band des Kapitals (2012: 60-61, 150-153), ist notwendigerweise darauf angewiesen, die Produktion und Zirkulation von Waren, Kapital und Informationen beständig zu beschleunigen. Vor diesem Hintergrund verwundert es kaum, dass die Logistik zu dem wurde, was sie laut Cowen heute ist: „not only the ,umbrella science of business management but [...], perhaps the central discipline of the contemporary world“" (S. 4). Daraus folgt, dass Logistik weitaus breiter und komplexer gedacht werden muss als alltägliche Redensarten des ,bloßen“ Transports von A nach B nahelegen. Logistik, so Cowen, ist eben nicht nur die Verschiffung von Dingen von einem Ort zum anderen, sondern eine angewandte Wissenschaft, der es im Kern darum geht, den Fluss von Waren-, Menschen- und Datenströmen nicht nur zu optimieren und zu rationalisieren, sondern auch gegen Unwägbarkeiten oder gar gezielte Sabotageakte abzusichern. Ob wir wollen oder nicht, diese Logiken greifen tiefer in unseren Alltag ein als es uns oft bewusst ist. Denn hinter jeder noch so banalen Bestellung, die wir bei Amazon, Ebay und Co. tätigen, tut sich ein komplexes, mehr oder weniger global koordiniertes Netzwerk aus menschlicher und maschineller Arbeit auf, von dem die allseits bekannte Paketübergabe an der Haustür nur den sichtbarsten aller Schritte darstellt. Wie Cowen schreibt: „The entire network of infrastructures, technologies, spaces, workers, and violence that makes the circulation of stuff possible remains tucked out of sight for those who engage with logistics only as consumers" (S. 1). Logistik, so könnte man es im Anschluss an David Harvey auch sagen (vgl. Toscano/ Kinkle 2015: 179), ist ein bisschen wie das Kapital selbst: meistens unsichtbar und dennoch (oder gerade deswegen) ungemein effizient. 
Wenn es in der Logistik einerseits um möglichst reibungsfreie Ströme von Waren, Arbeitskräften und Gütern geht - um jene „infrastructures, technologies, institutions, labor forces, and regulations that support the building of the seamless' corridors and gateways of logistics space" (S. 19) -, dann rücken andererseits auch jene Störfälle in den Vordergrund, die den streng getakteten Abläufen der Logistik- und Transportindustrie sehr schnell einen Strich durch die Rechnung machen: technische Defekte, Lieferengpässe, Umweltkatastrophen und, nicht zuletzt, Arbeitsstreiks, Sabotageakte und Blockaden. Genau hier zeigt sich die enorme stadtgeographische Relevanz von Cowens Untersuchung. Um den Faden des obigen Beispiels wiederaufzunehmen: Nicht zuletzt der Boom des Internet-Versandhandels bringt städtische Infrastrukturen und Verkehrsnetzwerke an ihre Grenzen. Die Gleichung ist einfach: Je größer das Bestellvolumen, das bei Logistikriesen wie Amazon umgeschlagen wird, desto höher ist auch das daraus resultierende Verkehrsaufkommen in Städten und somit das Risiko von Engpässen, die einer effizienten Warenauslieferung im Wege stehen:

„Der Aufstieg des Internethandels stellt neuartige Verteilungsherausforderungen an Handels- und Logistikunternehmen, sowohl was das Auslieferungsvolumen als auch was die Komplexität der Verteilung betrifft [...]. Das drängende Problem jeglicher stadt-logistischer Projekte ist eindeutig: Städte werden von Kräften beherrscht, die reibungslosen Abläufen zuwiderlaufen." (S. 180; Übers. F. N.)

Mit anderen Worten: Der Drang nach immer kürzeren Liefer- und Umschlagzeiten bleibt keineswegs abstrakt, sondern schreibt sich tief in die materielle Bausubstanz urbaner Räume ein. Cowens exemplarisches Beispiel: Dubai Logistics City, eine aus dem Boden gestampfte logistische ,Modellstadt', deren gebaute Räumlichkeit die Widersprüche logistischen Städtebaus schon jetzt auf den Punkt bringt:

„Auf der einen Seite ist der Bau einer logistischen Stadt ein wichtiges Ereignis für jene Produktion von urbanem Raum und urbaner Infrastruktur, die vollkommen darauf ausgerichtet ist, die Ströme globaler Lieferketten abzustützen. Auf der anderen Seite birgt der Anspruch, diese Ströme vor Unterbrechungen abzusichern, aber auch neue Formen der politisch-geographischen Einhegung [enclosure]. Für eine Stadt, die sich voll und ganz der Logistik verschreibt, führt die Störung von logistischen Abläufen zu Systemverwundbarkeit. Kräfte, die sich diesen Abläufen entgegensetzen, werden als Sicherheitsbedrohungen gehandhabt." (S. 173; Übers. F. N.)

Was sich hier abzeichnet, sind die groben Umrisse einer logistischen Stadt, deren Funktionswege zunehmend auf die ökonomischen, politischen und sozialräumlichen Erfordernisse globaler Kapitalströme zugeschnitten sind. Nicht nur findet Cowen vielfältige Beispiele für die logistische Umgestaltung urbaner Räume weltweit, auch bietet sie mit dem Konzept der logistischen Stadt eine plausible theoretische Erklärung für ebendiese Veränderungen an.[2] Für die kritische Stadtgeographie besonders interessant: Cowen liefert mit ihrer Deutung der logistischen Stadt vielleicht sogar so etwas wie die materielle Kehrseite der ,Smart City', die in der kritischen Stadtgeographie 
bislang - zwar nicht ausschließlich, aber doch vorrangig - als ,Smart City6Diskurs verhandelt wurde (etwa Bauriedl/Strüver 2017). Cowens Buch erweitert hier das Blickfeld erheblich, indem es eine städtische Realität entwirft, in der die ,smarte'Vernetzung, Abschöpfung und Inwertsetzung von Daten nur Teilaspekte einer breiteren - im Großen und Ganzen: logistischen - Umwälzung darstellen. Die ,logistics city als materieller Unterbau der ,Smart City‘? Es könnte eine lohnende Aufgabe empirischer und kritischer Stadtforschung sein, diese These zu untermauern, weiterzudenken oder, falls nötig, auch wieder zu verwerfen.

\section{Endnoten}

[1] Dass die gewaltvolle Geschichte der Logistik bis zu den Schiffsrouten des transatlantischen Sklavenhandels zurückreicht, legen wiederum Stefano Harney und Fred Moten (2013: 87-99) eindrucksvoll dar.

[2] Womit keineswegs gesagt sein soll, dass Cowen den Begriff der logistischen Stadt zuerst benutzte oder gar ,erfand".

\section{Autor innen}

Fabian Namberger studierte Politikwissenschaft und Soziologie. Er promoviert zur politischen Ökonomie von selbstfahrenden Fahrzeugen und urbaner Logistik.

fabian.namberger@gold.ac.uk

\section{Literatur}

Bauriedl, Sybille / Strüver, Anke (2017): Smarte Städte. Digitalisierte urbane Infrastrukturen und ihre Subjekte als Themenfeld kritischer Stadtforschung. In: sub \urban, 5/1, 87-104.

Bernes, Jasper (2013): Logistics, counterlogistics and the communist prospect. In: Endnotes 3 . https://endnotes.org.uk/issues/3/en/jasper-bernes-logistics-counterlogistics-and-thecommunist-prospect (letzter Zugriff am 21.4.2018).

Cowen, Deborah (2014): The Deadly Life of Logistics. Mapping Violence in Global Trade. Minneapolis: University of Minnesota Press.

Danyluk, Martin (2017): Capital's logistical fix. Accumulation, globalization, and the survival of capitalism. In: Environment and Planning D: Society and Space. https://doi. org/10.1177/0263775817703663 (letzter Zugriff am 10.3.2018).

Fisher, Mark (2013): Kapitalistischer Realismus ohne Alternative? Hamburg: VSA.

Gregson, Nicky / Crang, Mike / Antonopoulos, Constantinos (2017): Holding together logistical worlds. Friction, seams and circulation in the emerging,global warehouse'. In: Environment and Planning D: Society and Space 35/3, 381-398.

Harney, Stefano / Moten, Fred (2013): The undercommons. Fugitive planning \& Black study. Wivenhoe: Minor Compositions.

Marx, Karl (2012 [1885]): Das Kapital. Kritik der politischen Ökonomie. Zweiter Band. Der Zirkulationsprozess des Kapitals. Berlin: Dietz.

Moody, Kim (2017): On New Terrain. How Capital is Shaping the Battleground of Class War. Chicago: Haymarket Books.

Morozov, Evgeny / Bria, Francesca (2017): Die Smarte Stadt neu denken. Wie urbane Technologien demokratisiert werden können. Berlin: Rosa Luxemburg Stiftung.

Söderström, Ola / Paasche, Till / Klauser, Francisco (2014): Smart cities as corporate storytelling. In: City 18/3, 307-320.

Sundarakani, Balan (2017): Transforming Dubai logistics corridor into a global logistics hub. In: Asian Journal of Management Cases 14/2, 115-136. 
The Invisible Committee (2007): The coming insurrection. https://theanarchistlibrary.org/ library/comite-invisible-the-coming-insurrection (letzter Zugriff am 10.3.2018).

The Invisible Committee (2014): To our friends. https://theanarchistlibrary.org/library/ the-invisible-committe-to-our-friends (letzter Zugriff am 10.3.2018).

Toscano, Alberto (2011): Logistics and opposition. http://www.metamute.org/editorial/ articles/logistics-and-opposition (letzter Zugriff am 10.3.2018).

Toscano, Alberto (2014): Lineaments of the logistical state. https://www.viewpointmag. com/2014/o9/28/lineaments-of-the-logistical-state/\#fn1-3310 (letzter Zugriff am 10.3.2018).

Toscano, Alberto / Kinkle Jeff (2015): Cartographies of the Absolute. Winchester/ Washington: Zero Books.

Transnational Social Strike Platform (2017): Logistics and the transnational social strike.

https://www.transnational-strike.info/2017/09/18/logistics-and-the-transnational-socialstrike-tss-journal-fall-2017-2/ (letzter Zugriff am 23.3.2018). 